\title{
An Introduction to the Theory of Imprecise Soft Sets
}

\author{
Tridiv Jyoti Neog \\ Dept. of Mathematics, CMJ University, Shillong, Meghalaya, India \\ tridivjyoti@gmail.com \\ Dusmanta Kumar Sut \\ Dept. of Mathematics, N N Saikia College, Titabor, Jorhat, India \\ sutdk001@yahoo.com
}

\begin{abstract}
This paper aims to introduce the theory of imprecise soft sets which is a hybrid model of soft sets and imprecise sets. It has been established that two independent laws of randomness are necessary and sufficient to define a law of fuzziness. Further, in case of fuzzy sets, the set theoretic axioms of exclusion and contradiction are not satisfied. Accordingly, the theory of imprecise sets has been developed where these mistakes arising in the literature of fuzzy sets are absent. Our work is an endeavor to combine imprecise sets with soft sets resulting in imprecise soft sets. We have put forward a matrix representation of imprecise soft sets. Finally we have studied the notion of similarity of two imprecise soft sets and put forward an application of similarity in a decision problem.
\end{abstract}

Index Terms - Imprecise Sets, Partial Presence, Soft Sets, Imprecise Soft Sets, Presence Level Matrix, Similarity of Imprecise Soft Sets

\section{Introduction}

The discovery of fuzzy sets by Zadeh in 1965 was a paradigm change in the history of mathematics. In the theory of fuzzy sets, it has been observed that the operation of complementation of a normal fuzzy set does not explain the principles of exclusion and contradiction followed by the classical sets. This is due to the fact that in the Zadehian definition of complementation, membership value and membership function had been taken to be of the same meaning [1]. In order to link fuzziness with probability, Zadeh then forwarded Probability-Possibility Consistency Principle. But no consistency between probability and fuzziness was reflected by that principle and two more Probability-Possibility Consistency Principles were forwarded thereafter by others. Recently Baruah [2] has introduced the theory of imprecise sets where these two mistakes in the literature of fuzzy sets are absent.

Most of the concepts we meet in our day to day life are vague in nature. Mathematical modeling of such day to day problems involving uncertainties is of great importance now a days. There are theories e.g. Probability Theory, Fuzzy Set Theory, Intuitionistic Fuzzy Set Theory, Rough Set Theory etc. to deal with uncertainties. However these theories have their own difficulties. Infact, the inadequacy of the parameterization tool do not allow these theories to handle vagueness properly. In 1999 Molodtsov [3] introduced the novel concept of Soft Sets and established the fundamental results of the new theory. Soft Set Theory is free from parameterization inadequacy syndrome of Fuzzy Set Theory, Rough Set Theory, Probability Theory etc. Maji [4] studied the theory of soft sets and initiated some new results. In 2011, Neog and Sut [5] put forward a new definition of complement of a soft set and showed that the axioms of exclusion and contradiction are satisfied by the soft sets also.

In recent times, researches have contributed a lot towards fuzzification of Soft Set Theory. Combining fuzzy sets and soft sets, Maji et al. [6] put forward a new model known as fuzzy soft set. He introduced some properties regarding fuzzy soft union, intersection, complement of a fuzzy soft set, De Morgan Law etc. These results were further revised and improved by Ahmad and Kharal [7]. They defined arbitrary fuzzy soft union and intersection and proved De Morgan Inclusions and De Morgan Laws in Fuzzy Soft Set Theory. Neog and Sut [8,9] have studied the theory of fuzzy soft sets in a new perspective and initiated several results related to fuzzy soft sets. In their work, Zadehian fuzzy sets have been replaced with the extended fuzzy sets initiated by Baruah.

The content of the paper is organized as follows: In section II, some preliminary concepts related to extended fuzzy sets, imprecise sets and soft sets have been put forward. Section III deals with the notion of imprecise soft set theory and some fundamental results. In section IV, a matrix representation for a total imprecise soft set has been developed and finally in section $\mathrm{V}$, the notion of similarity of two total imprecise soft sets has been put forward and the proposed notion has been applied in a medical diagnosis problem. 


\section{Preliminaries}

In this section, we first recall some concepts and definitions which would be needed in the sequel. The following notions regarding extended definition of fuzzy sets and imprecise sets are due to Baruah ([1],[2],[10],[11],[12]).

\subsection{Extended Definition of Union and Intersection of Fuzzy Sets}

$$
\text { Let } \begin{aligned}
A\left(\mu_{1}, \mu_{2}\right) & =\left\{x, \mu_{1}(x), \mu_{2}(x) ; x \in U\right\} \text { and } \\
B\left(\mu_{3}, \mu_{4}\right) & =\left\{x, \mu_{3}(x), \mu_{4}(x) ; x \in U\right\}
\end{aligned}
$$

be two fuzzy sets defined over the same universe $U$. Then the operations intersection and union are defined as

$$
\begin{aligned}
& A\left(\mu_{1}, \mu_{2}\right) \cap B\left(\mu_{3}, \mu_{4}\right) \\
& =\left\{x, \min \left(\mu_{1}(x), \mu_{3}(x)\right), \max \left(\mu_{2}(x), \mu_{4}(x)\right) ; x \in U\right\}
\end{aligned}
$$

and

$$
\begin{aligned}
& A\left(\mu_{1}, \mu_{2}\right) \cup B\left(\mu_{3}, \mu_{4}\right) \\
& =\left\{x, \max \left(\mu_{1}(x), \mu_{3}(x)\right), \min \left(\mu_{2}(x), \mu_{4}(x)\right) ; x \in U\right\} .
\end{aligned}
$$

\subsection{Complement of a Fuzzy Set Using Extended Definition}

For usual fuzzy sets $A(\mu, 0)=\{x, \mu(x), 0 ; x \in U\}$ and $B(1, \mu)=\{x, 1, \mu(x) ; x \in U\}$ defined over the same universe $U$, we have

$$
\begin{aligned}
& A(\mu, 0) \cap B(1, \mu) \\
& =\{x, \min (\mu(x), 1), \max (0, \mu(x)) ; x \in U\} \\
& =\{x, \mu(x), \mu(x) ; x \in U\}, \text { which is nothing but the null } \\
& \operatorname{set} \varphi \text { and }
\end{aligned}
$$$$
A(\mu, 0) \cup B(1, \mu)
$$$$
=\{x, \max (\mu(x), 1), \min (0, \mu(x)) ; x \in U\}
$$$$
=\{x, 1,0 ; x \in U\} \text {, which is nothing but the universal set }
$$
$U$.

This means if we define a fuzzy set $(A(\mu, 0))^{c}=\{x, 1, \mu(x) ; x \in U\}$, it is nothing but the complement of $A(\mu, 0)=\{x, \mu(x), 0 ; x \in U\}$.

\subsection{Imprecise Number}

An imprecise number $[\alpha, \beta, \gamma]$ is an interval around the real number $\beta$ with the elements in the interval being partially present.

\subsection{Partial Presence}

Partial presence of an element in an imprecise real number $[\alpha, \beta, \gamma]$ is described by the presence level indicator function $p(x)$ which is counted from the reference function $r(x)$ such that the presence level for any $\quad x, \quad \alpha \leq x \leq \gamma \quad$ is $(p(x) \quad-\quad r(x))$, where $0 \leq r(x) \leq p(x) \leq 1$.

\subsection{Normal Imprecise Number}

A normal imprecise number $N=[\alpha, \beta, \gamma]$ is associated with a presence level indicator function $\mu_{N}(x)$, where

$$
\mu_{N}(x)=\left\{\begin{array}{l}
\psi_{1}(x), \text { if } \alpha \leq x \leq \beta \\
\psi_{2}(x), \text { if } \beta \leq x \leq \gamma \\
0, \text { otherwise }
\end{array}\right.
$$

with a constant reference function 0 in the entire real line. Here $\psi_{1}(x)$ is continuous and non-decreasing in the interval $[\alpha, \beta]$ and $\psi_{2}(x)$ is continuous and nonincreasing in the interval $[\beta, \gamma]$, with

$$
\begin{aligned}
& \psi_{1}(\alpha)=\psi_{2}(\gamma)=0 \\
& \psi_{1}(\beta)=\psi_{2}(\beta)=1
\end{aligned}
$$

Here, the imprecise number would be characterized by $\left\{x, \mu_{N}(x), 0: x \in R\right\}, R$ being the real line.

\subsection{Distribution Function and Complementary Distribution Function}

For a normal imprecise number $N=[\alpha, \beta, \gamma]$ with a presence level indicator function $\mu_{N}(x)$, where

$$
\mu_{N}(x)=\left\{\begin{array}{l}
\psi_{1}(x), \text { if } \alpha \leq x \leq \beta \\
\psi_{2}(x), \text { if } \beta \leq x \leq \gamma \\
0, \text { otherwise }
\end{array}\right.
$$

such that

$$
\begin{aligned}
& \psi_{1}(\alpha)=\psi_{2}(\gamma)=0, \\
& \psi_{1}(\beta)=\psi_{2}(\beta)=1 .
\end{aligned}
$$

with constant reference function equal to $0, \psi_{1}(x)$ is the distribution function of a random variable defined in the interval $[\alpha, \beta]$ and $\psi_{2}(x)$ is the complementary distribution function of another random variable defined in the interval $[\beta, \gamma]$.

We are using the term random variable here in the broader measure theoretic sense which does not require that the notion of probability need to appear in defining randomness.

\subsection{Complement of a Normal Imprecise Number}

For a normal imprecise number $N=\left\{x, \mu_{N}(x), 0: x \in R\right\} \quad, \quad$ the complement $N^{c}=\left\{x, 1, \mu_{N}(x): x \in R\right\}$ will have constant presence 
level indicator function equal to 1 , the reference function being $\mu_{N}(x)$ for $-\infty<x<\infty$.

\subsection{Complement of an Imprecise Set}

If a normal imprecise number $N=[\alpha, \beta, \gamma]$ is defined with a presence level indicator function $\mu_{N}(x)$, where

$$
\mu_{N}(x)=\left\{\begin{array}{l}
\psi_{1}(x), \text { if } \alpha \leq x \leq \beta \\
\psi_{2}(x), \text { if } \beta \leq x \leq \gamma \\
0, \text { otherwise }
\end{array}\right.
$$

With

$$
\begin{aligned}
& \psi_{1}(\alpha)=\psi_{2}(\gamma)=0, \\
& \psi_{1}(\beta)=\psi_{2}(\beta)=1 .
\end{aligned}
$$

the complement $N^{C}$ should have the presence level indicator function $\mu_{N^{c}}(x)$, with $\mu_{N^{c}}(x)=1,-\infty<x<\infty$, where $\mu_{N^{c}}(x)$ is to be counted from $\psi_{1}(x)$ if $\alpha \leq x \leq \beta$, from $\psi_{2}(x)$ if $\beta \leq x \leq \gamma$, and from 0 , otherwise.

Molodtsov [3]defined soft set in the following way-

\subsection{Soft Set}

A pair $(F, E)$ is called a soft set (over $U$ ) if and only if $F$ is a mapping of $E$ into the set of all subsets of the set $U$.

In other words, the soft set is a parameterized family of subsets of the set $U$. Every set $F(\varepsilon), \varepsilon \in E$, from this family may be considered as the set of $\varepsilon$ - elements of the soft set $(F, E)$, or as the set of $\varepsilon$ - approximate elements of the soft set.

\section{An Introduction To The Theory of Imprecise Soft Sets}

\subsection{Imprecise Soft Set}

Let $U$ be the initial universe and $E$ be the set of parameters. Let $A \subseteq E$ and $\tilde{P}(U)$ denote the set of all imprecise subsets over the universe $U$. Then a pair $(F, A)$ is called an imprecise soft set over $U$ where $F: A \rightarrow \tilde{P}(U)$ is a mapping from $A$ into $\tilde{P}(U)$.

\subsection{Example}

Let $U=\left\{c_{1}, c_{2}, c_{3}, c_{4}\right\}$ be the set of four cars under consideration and

$$
E=\left\{\begin{array}{l}
e_{1}(\text { costly }), e_{2}(\text { Beautiful }), e_{3}(\text { Fuel Efficient }), \\
\left.e_{4} \text { (ModernTech nology }\right), e_{5} \text { (Luxurious) }
\end{array}\right\}
$$

be the set of parameters and $A=\left\{e_{1}, e_{2}, e_{3}\right\} \subseteq E$. Then $(F, A)$

$$
\begin{gathered}
=\left\{F\left(e_{1}\right)=\left\{\left(c_{1}, 0.3,0\right),\left(c_{2}, 0.4,0\right),\left(c_{3}, 0.2,0\right),\left(c_{4}, 0.7,0\right)\right\},\right. \\
F\left(e_{2}\right)=\left\{\left(c_{1}, 0.6,0\right),\left(c_{2}, 0.7,0\right),\left(c_{3}, 0.3,0\right),\left(c_{4}, 0.5,0\right)\right\}, \\
\left.F\left(e_{3}\right)=\left\{\left(c_{1}, 0.1,0\right),\left(c_{2}, 0.2,0\right),\left(c_{3}, 0.1,0\right),\left(c_{4}, 0.6,0\right)\right\}\right\}
\end{gathered}
$$

is the imprecise soft set representing the 'attractiveness of the car' which Mr. $X$ is going to buy.

\subsection{Total Imprecise Soft Set}

Let $U$ be the initial universe, $E$ be the set of parameters and $\tilde{P}(U)$ denote the set of all imprecise subsets over the universe $U$. Then the pair $(F, E)$ is called a total imprecise soft set over $U$ where $F: E \rightarrow \tilde{P}(U)$ is a mapping from $E$ into $\tilde{P}(U)$.

\subsection{Null Imprecise Soft Set}

An imprecise soft set $(F, A)$ over $U$ is said to be null imprecise soft set (with respect to the parameter set $A$ ), denoted by $(\tilde{\varphi}, A)$ if $\forall \varepsilon \in A, F(\varepsilon)$ is the null set $\varphi$. The Null Imprecise Soft Set is not unique, it depends upon the set of parameters under consideration.

\subsection{Absolute Imprecise Soft Set}

An imprecise soft set $(F, A)$ over $U$ is said to be absolute imprecise soft set (with respect to the parameter set $A$ ), denoted by $(\tilde{U}, A)$ if $\forall \varepsilon \in A, F(\varepsilon)$ is the absolute set $U$. The Absolute Imprecise Soft Set is not unique, it depends upon the set of parameters under consideration.

\subsection{Union of Imprecise Soft Sets}

Union of two imprecise soft sets $(F, A)$ and $(G, B)$ over $(U, E)$ is an imprecise soft set $(H, C)$ where $C=A \cup B$ and $\forall \varepsilon \in C$,

$$
H(\varepsilon)=\left\{\begin{array}{l}
F(\varepsilon), \text { if } \varepsilon \in A-B \\
G(\varepsilon), \text { if } \varepsilon \in B-A \\
F(\varepsilon) \cup G(\varepsilon), \text { if } \varepsilon \in A \cap B
\end{array}\right.
$$

and is written as $(F, A) \tilde{\cup}(G, B)=(H, C)$.

\subsection{Intersection of Imprecise Soft Sets}

Let $(F, A)$ and $(G, B)$ be two imprecise soft sets over $(U, E)$ with $A \cap B \neq \phi$. Then intersection of the imprecise soft sets $(F, A)$ and $(G, B)$ is an imprecise soft set $(H, C)$ where $C=A \cap B$ and $\forall \varepsilon \in C$, $H(\varepsilon)=F(\varepsilon) \cap G(\varepsilon)$.

$$
\text { We write }(F, A) \tilde{\cap}(G, B)=(H, C) \text {. }
$$

\subsection{Imprecise Soft Subset}

For two imprecise soft sets $(F, A)$ and $(G, B)$ over $(U, E)$, we say that $(F, A)$ is an imprecise soft subset of $(G, B)$, if 
(i) $A \subseteq B$

(ii) For all $\varepsilon \in A, F(\varepsilon) \subseteq G(\varepsilon)$ and is written as $(F, A) \simeq(G, B)$.

\subsection{Equality of Two Imprecise Soft Sets}

For two imprecise soft sets $(F, A)$ and $(G, B)$ over $(U, E)$, we say that $(F, A)$ is equal to $(G, B)$, if $(F, A)$ $\tilde{\subseteq}(G, B)$ and $(G, B) \widetilde{\subseteq}(F, A)$.

\subsection{Complement of an Imprecise Soft Set}

The complement of an imprecise soft set $(F, A)$ is denoted by $(F, A)^{c}$ and is defined by $(F, A)^{c}=\left(F^{c}, A\right)$ where $F^{c}: A \rightarrow \tilde{P}(U)$ is a mapping given by $F^{c}(\varepsilon)=(F(\varepsilon))^{c}, \forall \varepsilon \in A$.

In other words,

$$
\begin{aligned}
& \forall \varepsilon \in A, \text { if } F(\varepsilon)=\left\{x, \mu_{F(\varepsilon)}(x), 0 ; x \in U\right\} \text {, then } \\
& F^{c}(\varepsilon)=\left\{x, 1, \mu_{F(\varepsilon)}(x) ; x \in U\right\} .
\end{aligned}
$$

\subsection{1 “AND" Operation of Two Imprecise Soft Sets}

If $(F, A)$ and $(G, B)$ be two imprecise soft sets, then " $(F, A)$ AND $(G, B)$ " is an imprecise soft set denoted by $(F, A) \wedge(G, B)$ and is defined by

$$
\begin{aligned}
& (F, A) \wedge(G, B)=(H, A \times B), \text { where } \\
& H(\alpha, \beta)=F(\alpha) \cap G(\beta), \forall \alpha \in A \text { and } \forall \beta \in B, \text { where }
\end{aligned}
$$
$\cap$ is the operation intersection of two imprecise sets.

\subsection{2 “OR" Operation of Two Imprecise Soft Sets}

If $(F, A)$ and $(G, B)$ be two imprecise soft sets, then " $(F, A)$ OR $(G, B)$ " is an imprecise soft set denoted by $(F, A) \vee(G, B)$ and is defined by

$$
(F, A) \vee(G, B)=(O, A \times B), \text { where }
$$

$O(\alpha, \beta)=F(\alpha) \cup G(\beta), \forall \alpha \in A$ and $\forall \beta \in B$, where $\cup$ is the operation union of two imprecise sets.

\subsection{Proposition}

For an imprecise soft set $(F, A)$ over $U$, we have,

$$
\begin{aligned}
& \text { 1. }(F, A) \tilde{\cup}(F, A)^{c}=(\tilde{U}, A) \\
& \text { 2. }(F, A) \tilde{\cap}(F, A)^{c}=(\tilde{\varphi}, A)
\end{aligned}
$$

\section{Proof}

1. Let $(F, A) \tilde{\cup}(F, A)^{c}=(F, A) \tilde{\cup}\left(F^{c}, A\right)=(H, A)$, where $\forall \varepsilon \in A$,

$$
\begin{aligned}
H(\varepsilon) & =F(\varepsilon) \cup F^{c}(\varepsilon) \\
& =F(\varepsilon) \cup(F(\varepsilon))^{c}
\end{aligned}
$$

$$
\begin{aligned}
& =\left\{x, \mu_{F(\varepsilon)}(x), 0 ; x \in U\right\} \cup\left\{x, 1, \mu_{F(\varepsilon)}(x) ; x \in U\right\} \\
& =\left\{x, \max \left(\mu_{F(\varepsilon)}(x), 1\right), \min \left(0, \mu_{F(\varepsilon)}(x)\right) ; x \in U\right\} \\
& =\{x, 1,0 ; x \in U\} \\
& =U
\end{aligned}
$$

Thus $(F, A) \tilde{\cup}(F, A)^{c}=(\tilde{U}, A)$

2. Let $(F, A) \tilde{\cap}(F, A)^{c}=(F, A) \tilde{\cap}\left(F^{c}, A\right)=(H, A)$, where $\forall \varepsilon \in A$,

$$
\begin{aligned}
H(\varepsilon) & =F(\varepsilon) \cap F^{c}(\varepsilon) \\
& =F(\varepsilon) \cup(F(\varepsilon))^{c} \\
& =\left\{x, \mu_{F(\varepsilon)}(x), 0 ; x \in U\right\} \cap\left\{x, 1, \mu_{F(\varepsilon)}(x) ; x \in U\right\} \\
& =\left\{x, \min \left(\mu_{F(\varepsilon)}(x), 1\right), \max \left(0, \mu_{F(\varepsilon)}(x)\right) ; x \in U\right\} \\
& =\left\{x, \mu_{F(\varepsilon)}(x), \mu_{F(\varepsilon)}(x) ; x \in U\right\} \\
& =\varphi
\end{aligned}
$$

Thus $(F, A) \tilde{\cap}(F, A)^{c}=(\tilde{\varphi}, A)$

\subsection{Proposition}

For imprecise soft sets $(F, A),(G, B)$ and $(H, C)$ over $(U, E)$, the following results are valid.

$$
\begin{aligned}
& \text { 1. }(\tilde{\varphi}, A)^{c}=(\tilde{U}, A) \\
& \text { 2. }(\tilde{U}, A)^{c}=(\tilde{\varphi}, A) \\
& \text { 3. }(F, A) \tilde{\cup}(\tilde{\varphi}, A)=(F, A)
\end{aligned}
$$

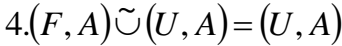

$$
\begin{aligned}
& \text { 5. }(F, A) \tilde{\cap}(\tilde{\varphi}, A)=(\tilde{\varphi}, A) \\
& \text { 6. }(F, A) \tilde{\cap}(\tilde{U}, A)=(F, A) \\
& \text { 7. }(F, A) \tilde{\cup}(\tilde{\varphi}, B)=(F, A) \text { if and only if } B \subseteq A \\
& \text { 8. }(F, A) \tilde{\cup}(U, B)=(U, B) \text { if and only if } A \subseteq B \\
& \text { 9. }(F, A) \tilde{\cap}(\tilde{\varphi}, B)=(\tilde{\varphi}, A \cap B) \\
& \text { 10. }(F, A) \tilde{\cap}(\tilde{U}, B)=(F, A \cap B) \\
& \text { 11.(i) }(F, A) \tilde{\cup}(G, B)=(G, B) \tilde{\cup}(F, A) \\
& \text { (ii) }(F, A) \tilde{\cap}(G, B)=(G, B) \tilde{\cap}(F, A) \\
& \text { 12.(i) }(F, A) \tilde{\cup}((G, B) \tilde{\cup}(H, C)) \\
& =((F, A) \tilde{\cup}(G, B)) \tilde{\cup}(H, C) \\
& \text { (ii) }(F, A) \tilde{\cap}((G, B) \tilde{\cap}(H, C)) \\
& =((F, A) \tilde{\cap}(G, B)) \tilde{\cap}(H, C) \\
& \text { 13.(i) }(F, A) \tilde{\cup}((G, B) \tilde{\cap}(H, C)) \\
& =((F, A) \tilde{\cup}(G, B)) \tilde{\cap}((F, A) \tilde{\cup}(H, C)) \\
& \text { (ii) }(F, A) \tilde{\cap}((G, B) \tilde{\cup}(H, C)) \\
& =((F, A) \tilde{\cap}(G, B)) \tilde{\cup}((F, A) \tilde{\cap}(H, C)) \\
& \text { 14.(i) }(F, A) \tilde{\cup}(F, A)=(F, A) \\
& \text { (ii) }(F, A) \tilde{\cap}(F, A)=(F, A) \\
& \text { 15. }\left((F, A)^{c}\right)^{c}=(F, A)
\end{aligned}
$$

Proof: The result immediately follows from definition. 
De Morgan Laws are not valid for imprecise soft sets with different sets of parameters. Instead, we have the following inclusions. De Morgan Laws are valid for imprecise soft sets with the same set of parameter.

\subsection{Proposition}

For imprecise soft sets $(F, A)$ and $(G, B)$ over $(U, E)$, one has the following:

$$
\begin{aligned}
& \text { 1. }(F, A)^{c} \tilde{\cap}(G, B)^{c} \tilde{\subseteq}((F, A) \tilde{\cup}(G, B))^{c} \\
& \text { 2. }((F, A) \tilde{\cap}(G, B))^{c} \tilde{\subseteq}(F, A)^{c} \tilde{\cup}(G, B)^{c}
\end{aligned}
$$

\section{Proof}

1. Let $(F, A) \tilde{\cup}(G, B)=(H, C)$, where $C=A \cup B$ and $\forall \varepsilon \in C$,

$H(\varepsilon)$

$$
=\left\{\begin{array}{l}
\left\{\left(x, \mu_{F(\varepsilon)}(x), 0\right): x \in U\right\}, \text { if } \varepsilon \in A-B \\
\left\{\left(x, \mu_{G(\varepsilon)}(x), 0\right): x \in U\right\}, \text { if } \varepsilon \in B-A \\
\left\{\left(x, \max \left(\mu_{F(\varepsilon)}(x), \mu_{G(\varepsilon)}(x)\right), 0\right): x \in U\right\}, \text { if } \varepsilon \in A \cap B
\end{array}\right.
$$

Thus $((F, A) \tilde{\cup}(G, B))^{c}=(H, C)^{c}=\left(H^{c}, C\right)$, where $C=A \cup B$ and $\forall \varepsilon \in C$,

$$
H^{c}(\varepsilon)
$$

$$
=(H(\varepsilon))^{c}
$$$$
=\left\{\begin{array}{l}
\left\{\left(x, 1, \mu_{F(\varepsilon)}(x)\right): x \in U\right\}, \text { if } \varepsilon \in A-B \\
\left\{\left(x, 1, \mu_{G(\varepsilon)}(x)\right): x \in U\right\}, \text { if } \varepsilon \in B-A \\
\left\{\left(x, 1, \max \left(\mu_{F(\varepsilon)}(x), \mu_{G(\varepsilon)}(x)\right)\right): x \in U\right\}, \text { if } \varepsilon \in A \cap B
\end{array}\right.
$$

Again, $(F, A)^{c} \tilde{\cap}(G, B)^{c}=\left(F^{c}, A\right) \tilde{\cap}\left(G^{c}, B\right)=(I, J)$, say, where $J=A \cap B$ and $\forall \varepsilon \in J$,

$I(\varepsilon)$

$=F^{c}(\varepsilon) \cap G^{c}(\varepsilon)$

$=\left\{\left(x, 1, \mu_{F(\varepsilon)}(x)\right): x \in U\right\} \cap\left\{\left(x, 1, \mu_{G(\varepsilon)}(x)\right): x \in U\right\}$

$=\left\{\left(x, 1, \max \left(\mu_{F(\varepsilon)}(x), \mu_{G(\varepsilon)}(x)\right)\right): x \in U\right\}$

We see that $J \subseteq C$ and $\forall \varepsilon \in J, I(\varepsilon)=H^{c}(\varepsilon)$

Thus $(F, A)^{c} \tilde{\cap}(G, B)^{c} \tilde{\subseteq}((F, A) \tilde{\cup}(G, B))^{c}$

It follows immediately that

$$
(F, A)^{c} \tilde{\cap}(G, B)^{c} \widetilde{\subseteq}((F, A) \tilde{\cup}(G, B))^{c}
$$

2. Let $(F, A) \tilde{\cap}(G, B)=(H, C)$, where $C=A \cap B$ and $\forall \varepsilon \in C$,

$H(\varepsilon)$

$=F(\varepsilon) \cap G(\varepsilon)$ $=\left\{\left(x, \mu_{F(\varepsilon)}(x), 0\right): x \in U\right\} \cap\left\{\left(x, \mu_{G(\varepsilon)}(x), 0\right): x \in U\right\}$

$=\left\{\left(x, \min \left(\mu_{F(\varepsilon)}(x), \mu_{G(\varepsilon)}(x)\right), 0\right): x \in U\right\}$

Thus $((F, A) \tilde{\cap}(G, B))^{c}=(H, C)^{c}=\left(H^{c}, C\right)$, where $C=A \cap B$ and $\forall \varepsilon \in C$,

$H^{c}(\varepsilon)$

$=(F(\varepsilon) \cap G(\varepsilon))^{c}$

$=\left\{\left(x, \min \left(\mu_{F(\varepsilon)}(x), \mu_{G(\varepsilon)}(x)\right), 0\right): x \in U\right\}$

$=\left\{\left(x, 1, \min \left(\mu_{F(\varepsilon)}(x), \mu_{G(\varepsilon)}(x)\right), 0\right): x \in U\right\}$

Again, $(F, A)^{c} \tilde{\cup}(G, B)^{c}=\left(F^{c}, A\right) \tilde{\cup}\left(G^{c}, B\right)=(I, J)$, say, where $J=A \cup B$ and $\forall \varepsilon \in J$,

$I(\varepsilon)$

$$
=\left\{\begin{array}{l}
\left\{\left(x, 1, \mu_{F(\varepsilon)}(x)\right): x \in U\right\}, \text { if } \varepsilon \in A-B \\
\left\{\left(x, 1, \mu_{G(\varepsilon)}(x)\right): x \in U\right\}, \text { if } \varepsilon \in B-A \\
\left\{\left(\left(x, 1, \min \left(\mu_{F(\varepsilon)}(x), \mu_{G(\varepsilon)}(x)\right)\right): x \in U\right\}, \text { if } \varepsilon \in A \cap B\right.
\end{array}\right.
$$

We see that $C \subseteq J$ and $\forall \varepsilon \in C, H^{c}(\varepsilon)=I(\varepsilon)$

It follows that $((F, A) \tilde{\cap}(G, B))^{c} \tilde{\subseteq}(F, A)^{c} \tilde{\cup}(G, B)^{c}$

\subsection{Proposition (De Morgan Laws)}

For imprecise soft sets $(F, A)$ and $(G, A)$ over $(U, E)$, one has the following:

1. $((F, A) \tilde{\cup}(G, A))^{c}=(F, A)^{c} \tilde{\cap}(G, A)^{c}$

2. $((F, A) \tilde{\cap}(G, A))^{c}=(F, A)^{c} \tilde{\cup}(G, A)^{c}$

\section{Proof}

1. Let $(F, A) \tilde{\cup}(G, A)=(H, A)$, where $\forall \varepsilon \in A$,

$$
\begin{aligned}
H(\varepsilon) & =F(\varepsilon) \cup G(\varepsilon) \\
& =\left\{\left(x, \max \left(\mu_{F(\varepsilon)}(x), \mu_{G(\varepsilon)}(x)\right), 0\right): x \in U\right\}
\end{aligned}
$$

Thus $((F, A) \tilde{\cup}(G, A))^{c}=(H, A)^{c}=\left(H^{c}, A\right)$, where $\forall \varepsilon \in A$,

$$
\begin{aligned}
H^{c}(\varepsilon) & =(H(\varepsilon))^{c} \\
& =\left\{\left(x, 1, \max \left(\mu_{F(\varepsilon)}(x), \mu_{G(\varepsilon)}(x)\right)\right): x \in U\right\}
\end{aligned}
$$

Again, $(F, A)^{c} \tilde{\cap}(G, A)^{c}=\left(F^{c}, A\right) \tilde{\cap}\left(G^{c}, A\right)=(I, A)$, say, where $\forall \varepsilon \in A$,

$I(\varepsilon) \quad=F^{c}(\varepsilon) \cap G^{c}(\varepsilon)$

$$
=\left\{\left(x, 1, \max \left(\mu_{F(\varepsilon)}(x), \mu_{G(\varepsilon)}(x)\right)\right): x \in U\right\}
$$

Thus $((F, A) \tilde{\cup}(G, A))^{c}=(F, A)^{c} \tilde{\cap}(G, A)^{c}$

2. Let $(F, A) \tilde{\cap}(G, A)=(H, A)$, where $\forall \varepsilon \in A$, 


$$
\begin{aligned}
H(\varepsilon) & =F(\varepsilon) \cap G(\varepsilon) \\
& =\left\{\left(x, \min \left(\mu_{F(\varepsilon)}(x), \mu_{G(\varepsilon)}(x)\right), 0\right): x \in U\right\}
\end{aligned}
$$

Thus $((F, A) \tilde{\cap}(G, A))^{c}=(H, A)^{c}=\left(H^{c}, A\right)$, where $\forall \varepsilon \in A$,

$$
\begin{aligned}
& \begin{aligned}
H^{c}(\varepsilon) & =(H(\varepsilon))^{c} \\
& =\left\{\left(x, 1, \min \left(\mu_{F(\varepsilon)}(x), \mu_{G(\varepsilon)}(x)\right)\right): x \in U\right\}
\end{aligned} \\
& \text { Again, }(F, A)^{c} \tilde{\cup}(G, A)^{c} \quad=\left(F^{c}, A\right) \tilde{\cup}\left(G^{c}, A\right) \\
& =(I, A), \text { say, where } \forall \varepsilon \in A,
\end{aligned}
$$

$$
\begin{aligned}
I(\varepsilon) \quad & =F^{c}(\varepsilon) \cup G^{c}(\varepsilon) \\
& =\left\{\left(x, 1, \min \left(\mu_{F(\varepsilon)}(x), \mu_{G(\varepsilon)}(x)\right)\right): x \in U\right\}
\end{aligned}
$$

Thus $((F, A) \tilde{\cap}(G, A))^{c}=(F, A)^{c} \tilde{\cup}(G, A)^{c}$

\subsection{Proposition}

For imprecise soft sets $(F, A)$ and $(G, B)$ over $(U, E)$, one has the following:

1. $((F, A) \wedge(G, B))^{c}=(F, A)^{c} \vee(G, B)^{c}$

2. $((F, A) \vee(G, B))^{c}=(F, A)^{c} \wedge(G, B)^{c}$

\section{Proof}

1. Let $(F, A) \wedge(G, B)=(H, A \times B)$, where $\forall \alpha \in A$ and $\forall \beta \in B$

$$
\begin{aligned}
H(\alpha, \beta) & =F(\alpha) \cap G(\beta) \\
& =\left\{\left(x, \mu_{F(\alpha)}(x), 0\right): x \in U\right\} \cap\left\{\left(x, \mu_{G(\beta)}(x), 0\right): x \in U\right\} \\
& =\left\{\left(x, \min \left(\mu_{F(\alpha)}(x), \mu_{G(\beta)}(x)\right), 0\right): x \in U\right\}
\end{aligned}
$$

Thus $((F, A) \wedge(G, B))^{c}=(H, A \times B)^{c}=\left(H^{c}, A \times B\right)$, where $\forall(\alpha, \beta) \in A \times B$,

$$
\begin{aligned}
H^{c}(\alpha, \beta) & =(H(\alpha, \beta))^{c} \\
= & (F(\alpha) \cap G(\beta))^{c} \\
= & \left\{\left(x, \min \left(\mu_{F(\alpha)}(x), \mu_{G(\beta)}(x)\right), 0\right): x \in U\right\} \\
= & \left\{\left(x, 1, \min \left(\mu_{F(\alpha)}(x), \mu_{G(\beta)}(x)\right)\right): x \in U\right\}
\end{aligned}
$$

Let $(F, A)^{c} \vee(G, B)^{c} \quad=\left(F^{c}, A\right) \vee\left(G^{c}, B\right)$

$$
=(O, A \times B) \text {, }
$$

where

$$
\begin{aligned}
& \forall \alpha \in A \text { and } \forall \beta \in B \\
& \begin{aligned}
O(\alpha, \beta) & =F^{c}(\alpha) \cup G^{c}(\beta) \\
& =\left\{\left(x, 1, \mu_{F(\alpha)}(x)\right): x \in U\right\} \cup\left\{\left(x, 1, \mu_{G(\beta)}(x)\right): x \in U\right\} \\
& =\left\{\left(x, 1, \min \left(\mu_{F(\alpha)}(x), \mu_{G(\beta)}(x)\right)\right): x \in U\right\}
\end{aligned}
\end{aligned}
$$

Thus $((F, A) \wedge(G, B))^{c}=(F, A)^{c} \vee(G, B)^{c}$
2. Let $(F, A) \vee(G, B)=(H, A \times B)$, where $\forall \alpha \in A$ and $\forall \beta \in B$

$$
\begin{aligned}
H(\alpha, \beta) & =F(\alpha) \cup G(\beta) \\
& =\left\{\left(x, \mu_{F(\alpha)}(x), 0\right): x \in U\right\} \cup\left\{\left(x, \mu_{G(\beta)}(x), 0\right): x \in U\right\} \\
& =\left\{\left(x, \max \left(\mu_{F(\alpha)}(x), \mu_{G(\beta)}(x)\right), 0\right): x \in U\right\}
\end{aligned}
$$

Thus $((F, A) \vee(G, B))^{c}=(H, A \times B)^{c}=\left(H^{c}, A \times B\right)$, where $\forall(\alpha, \beta) \in A \times B$,

$$
\begin{aligned}
H^{c}(\alpha, \beta) & =(H(\alpha, \beta))^{c} \\
= & (F(\alpha) \cup G(\beta))^{c} \\
= & \left\{\left(x, \max \left(\mu_{F(\alpha)}(x), \mu_{G(\beta)}(x)\right), 0\right): x \in U\right\}^{c} \\
= & \left\{\left(x, 1, \max \left(\mu_{F(\alpha)}(x), \mu_{G(\beta)}(x)\right)\right): x \in U\right\}
\end{aligned}
$$

Let $(F, A)^{c} \wedge(G, B)^{c} \quad=\left(F^{c}, A\right) \wedge\left(G^{c}, B\right)$

$$
=(O, A \times B) \text {, }
$$

where $\forall \alpha \in A$ and $\forall \beta \in B$

$$
\begin{aligned}
O(\alpha, \beta) & =F^{c}(\alpha) \cap G^{c}(\beta) \\
& =\left\{\left(x, 1, \mu_{F(\alpha)}(x)\right): x \in U\right\} \cap\left\{\left(x, 1, \mu_{G(\beta)}(x)\right): x \in U\right\} \\
& =\left\{\left(x, 1, \max \left(\mu_{F(\alpha)}(x), \mu_{G(\beta)}(x)\right)\right): x \in U\right\}
\end{aligned}
$$

Thus $((F, A) \vee(G, B))^{c}=(F, A)^{c} \wedge(G, B)^{c}$.

\section{Matrix Representation of Total Imprecise Soft Set}

Let $U=\left\{c_{1}, c_{2}, c_{3}, \ldots \ldots \ldots \ldots \ldots \ldots \ldots c_{m}\right\}$ be the universal set and $E$ be the set of parameters given by

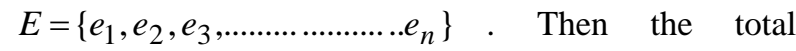
imprecise soft set $(F, E)$ can be expressed in matrix form as $\quad A=\left[a_{i j}\right]_{m \times n}$ or simply by $\left[a_{i j}\right], i=$

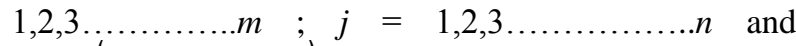
$a_{i j}=\left(p_{j}\left(c_{i}\right), r_{j}\left(c_{i}\right)\right) \quad ; \quad$ where $p_{j}\left(c_{i}\right)$ and $r_{j}\left(c_{i}\right)$ represent the presence level indicator function and the reference function respectively of $c_{i}$ in the imprecise set $F\left(e_{j}\right)$ so that $\delta_{i j}\left(c_{i}\right)=p_{j}\left(c_{i}\right)-r_{j}\left(c_{i}\right)$ gives the presence level of $c_{i}$. We shall identify a total imprecise soft set with its total imprecise soft matrix and use these two concepts interchangeable. The set of all $m \times n$ total imprecise soft matrices over $U$ will be denoted by TISM $_{m \times n}$.

\subsection{Presence Level Matrix of a Total Imprecise Soft Matrix}

We define the presence level matrix corresponding to the matrix $A$ as $P L(A)=\left[\delta_{(A) i j}\right]_{m \times n}$, where 
$\delta_{(A) i j}=p_{j}\left(c_{i}\right)-r_{j}\left(c_{i}\right) \quad \forall i=1,2,3 \ldots \ldots, m \quad$ and $j=1,2,3 \ldots . . ., n$, where $p_{j}\left(c_{i}\right)$ and $r_{j}\left(c_{i}\right)$ represent the presence level indicator function and the reference function respectively of $c_{i}$ in the imprecise set $F\left(e_{j}\right)$.

\subsection{Example}

Let $U=\left\{c_{1}, c_{2}, c_{3}, c_{4}\right\}$ be the universal set and $E$ be the set of parameters given by $E=\left\{e_{1}, e_{2}, e_{3}\right\}$. We consider a total imprecise soft set

$$
\begin{aligned}
(F, E) & \left\{F\left(e_{1}\right)=\left\{\left(c_{1}, 0.7,0\right),\left(c_{2}, 0.1,0\right),\left(c_{3}, 0.2,0\right),\left(c_{4}, 0.6,0\right)\right\},\right. \\
& F\left(e_{2}\right)=\left\{\left(c_{1}, 0.8,0\right),\left(c_{2}, 0.6,0\right),\left(c_{3}, 0.1,0\right),\left(c_{4}, 0.5,0\right)\right\}, \\
& \left.F\left(e_{3}\right)=\left\{\left(c_{1}, 0.1,0\right),\left(c_{2}, 0.2,0\right),\left(c_{3}, 0.7,0\right),\left(c_{4}, 0.3,0\right)\right\}\right\}
\end{aligned}
$$

We would represent this total imprecise soft set in matrix form as

$$
A=\left[a_{i j}\right]_{4 \times 3}=\left[\begin{array}{rrr}
(0.7,0) & (0.8,0) & (0.1,0) \\
(0.1,0) & (0.6,0) & (0.2,0) \\
(0.2,0) & (0.1,0) & (0.7,0) \\
(0.6,0) & (0.5,0) & (0.3,0)
\end{array}\right]_{4 \times 3}
$$

The presence level matrix corresponding to the matrix $\mathrm{A}$ is given by

$$
P L(A)=\left[\begin{array}{lll}
0.7 & 0.8 & 0.1 \\
0.1 & 0.6 & 0.2 \\
0.2 & 0.1 & 0.7 \\
0.6 & 0.5 & 0.3
\end{array}\right]
$$

\section{Similarity Between Two Total Imprecise Soft Sets And Its Application In Medical Diagnosis}

\subsection{Similarity Between Two Total Imprecise Soft Sets}

Let $(F, E)$ and $(G, E)$ be two total imprecise soft sets over $(U, E)$, where $U=\left\{c_{1}, c_{2}, c_{3}, \ldots \ldots \ldots \ldots \ldots \ldots c_{m}\right\}$ and

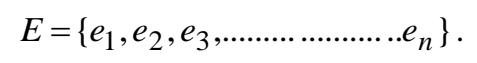

Let $(F, E) \tilde{\cup}(G, E)=(P, E)$

and $(F, E) \tilde{\cap}(G, E)=(Q, E)$.

We assume that $A=\left[a_{i j}\right]$ and $B=\left[b_{i j}\right]$ are the total imprecise soft matrices corresponding to $(P, E)$ and $(Q, E)$ respectively, where $a_{i j}=\left(p_{j}\left(c_{i}\right), r_{j}\left(c_{i}\right)\right)$ and $b_{i j}=\left(p^{\prime}{ }_{j}\left(c_{i}\right), r^{\prime}{ }_{j}\left(c_{i}\right)\right)$.

The Presence Level Matrices corresponding to the matrices $A$ and $B$ are respectively, $P L(A)=\left[\delta_{(A) i j}\right]$, where $\delta_{(A) i j}=p_{j}\left(c_{i}\right)-r_{j}\left(c_{i}\right)$ and $P L(B)=\left[\delta_{(B) i j}\right]$, where $\delta_{(B) i j}=p^{\prime}{ }_{j}\left(c_{i}\right)-r^{\prime}{ }_{j}\left(c_{i}\right)$.

Let $S((F, E),(G, E))$ denote the similarity between the total imprecise soft sets $(F, E)$ and $(G, E)$.

Let $S_{j}((F, E),(G, E))$ represent the similarity between the $e_{j}$ approximations $F\left(e_{j}\right)$ and $G\left(e_{j}\right) \forall e_{j}$. Then we define

$$
S_{j}((F, E),(G, E))=\frac{\sum_{i=1}^{m}\left(\delta_{(A) i j} \wedge \delta_{(B) i j}\right)}{\sum_{i=1}^{m}\left(\delta_{(A) i j} \vee \delta_{(B) i j}\right)} \text { and }
$$

$S((F, E),(G, E))$

$$
=\max _{j}\left\{S_{j}((F, E),(G, E))\right\}, j=1,2,3 \ldots \ldots \ldots n
$$

\subsection{Proposition}

Let $(F, E),(G, E)$ and $(H, E)$ be three total imprecise soft sets over $(U, E)$. Then the following results are valid.

$$
\begin{array}{ll}
\text { (i) } & S\left((F, E),(F, E)^{c}\right)=0 \\
\text { (ii) } & S((F, E),(G, E))=S((G, E),(F, E)) \\
\text { (iii) } & (F, E)=(G, E) \Rightarrow S((F, E),(G, E))=1 \\
\text { (iv) } & (F, E) \tilde{\cap}(G, E)=\tilde{\varphi} \Leftrightarrow S((F, E),(G, E))=0 \\
\text { (v) } & (F, E) \tilde{\subseteq}(H, E) \tilde{\subseteq}(G, E) \\
& \Rightarrow S((F, E),(G, E)) \leq S((H, E),(G, E))
\end{array}
$$

Proof: The proof is straight forward.

\subsection{Significantly Similar Total Imprecise Soft Sets}

Let $(F, E)$ and $(G, E)$ be two total imprecise soft sets over the same universe $(U, E)$. We call the two total imprecise soft sets to be significantly similar if $S((F, E),(G, E)) \geq \frac{1}{2}$

\subsection{Application of Similarity in Medical Diagnosis}

In this section, we will try to find out whether an ill person having certain symptoms is suffering from pneumonia or not. We first construct a model imprecise soft set for pneumonia and the imprecise soft set of symptoms for the ill persons. Next we find the similarity of these two sets. If they are significantly similar then we conclude that the person is possibly suffering from pneumonia.

Let our universal set contain only two elements 'yes (y)' and 'no $(n)$ ', i.e. $U=\{y, n\}$. Here the set of parameters $E$ is the set of certain visible symptoms. Let $E=\left\{e_{1}, e_{2}, e_{3}, e_{4}, e_{5}, e_{6}, e_{7}, e_{8}\right\}$, where $e_{1}=$ body temperature, $e_{2}=$ cough with chest congestion, $e_{3}=$ 
cough with no chest congestion, $e_{4}=$ body ache, $e_{5}=$ headache, $e_{6}=$ loose motion, $e_{7}=$ breathing trouble. Our model fuzzy soft set for pneumonia $(F, E)$ is given in Table 1 and this can be prepared with the help of a physician. In a similar fashion, we construct the imprecise soft sets corresponding to the two ill persons under consideration as given in Table 2, 3 respectively.

\section{Table 1:}

\section{Model Imprecise Soft Set for Pneumonia}

$\begin{array}{cccccccc}(F, E) & e_{1} & e_{2} & e_{3} & e_{4} & e_{5} & e_{6} & e_{7} \\ y & (1,0) & (1,0) & (0,0) & (1,0) & (0,0) & (0.0) & (1,0) \\ n & (0,0) & (0,0) & (1,0) & (0,0) & (1,0) & (1,0) & (0,0)\end{array}$

\section{Table 2:}

\section{Imprecise Soft Set for first ill person}

$\begin{array}{cccccccc}\left(F_{1}, E\right) & e_{1} & e_{2} & e_{3} & e_{4} & e_{5} & e_{6} & e_{7} \\ y & (0.2,0) & (0,0) & (0.6,0) & (0.3,0) & (0.5,0) & (0.1,0) & (0.1,0) \\ n & (0.1,0) & (0.3,0) & (0.2,0) & (0.1,0) & (0.3,0) & (0.5,0) & (0.6,0)\end{array}$

\section{Table 3:}

\section{Imprecise Soft Set for second ill person}

$\begin{array}{cccccccc}\left(F_{2}, E\right) & e_{1} & e_{2} & e_{3} & e_{4} & e_{5} & e_{6} & e_{7} \\ y & (0.7,0) & (0.9,0) & (0.2,0) & (0.6,0) & (0.5,0) & (0.1,0) & (0.8,0) \\ n & (0.1,0) & (0.1,0) & (0.2,0) & (0.1,0) & (0.3,0) & (0.7,0) & (0.1,0)\end{array}$

Case I: Similarity between $(F, E)$ and $\left(F_{1}, E\right)$

$$
(F, E) \tilde{\cup}\left(F_{1}, E\right)=\left(P_{1}, E\right)
$$

$\begin{array}{cccccccc}\left(P_{1}, E\right) & e_{1} & e_{2} & e_{3} & e_{4} & e_{5} & e_{6} & e_{7} \\ y & (1,0) & (1,0) & (0.6,0) & (1,0) & (0.5,0) & (0.1,0) & (1,0) \\ n & (0.1,0) & (0.3,0) & (1,0) & (0.1,0) & (1,0) & (1,0) & (0.6,0)\end{array}$

$$
(F, E) \tilde{\cap}\left(F_{1}, E\right)=\left(Q_{1}, E\right)
$$

$$
\begin{array}{cccccccc}
\left(Q_{1}, E\right) & e_{1} & e_{2} & e_{3} & e_{4} & e_{5} & e_{6} & e_{7} \\
y & (0.2,0) & (0,0) & (0,0) & (0.3,0) & (0,0) & (0,0) & (0.1,0) \\
n & (0,0) & (0,0) & (0.2,0) & (0,0) & (0.3,0) & (0.5,0) & (0,0)
\end{array}
$$

The Imprecise soft matrices corresponding to these two Imprecise soft sets $\left(P_{1}, E\right)$ and $\left(Q_{1}, E\right)$ are given by,

$$
A_{1}=\left[\begin{array}{ccccccc}
(1,0) & (1,0) & (0.6,0) & (1,0) & (0.5,0) & (0.1,0) & (1,0) \\
(0.1,0) & (0.3,0) & (1,0) & (0.1,0) & (1,0) & (1,0) & (0.6,0)
\end{array}\right]
$$

$$
B_{1}=\left[\begin{array}{ccccccc}
(0.2,0) & (0,0) & (0,0) & (0.3,0) & (0,0) & (0,0) & (0.1,0) \\
(0,0) & (0,0) & (0.2,0) & (0,0) & (0.3,0) & (0.5,0) & (0,0)
\end{array}\right]
$$

The corresponding presence level matrices are given by,

$$
\begin{gathered}
P L\left(A_{1}\right)=\left[\begin{array}{ccccccc}
1 & 1 & 0.6 & 1 & 0.5 & 0.1 & 1 \\
0.1 & 0.3 & 1 & 0.1 & 1 & 1 & 0.6
\end{array}\right] \\
P L\left(B_{1}\right)=\left[\begin{array}{ccccccc}
0.2 & 0 & 0 & 0.3 & 0 & 0 & 0.1 \\
0 & 0 & 0.2 & 0 & 0.3 & 0.5 & 0
\end{array}\right]
\end{gathered}
$$

Calculations give,

$S_{1}\left((F, E),\left(F_{1}, E\right)\right)=0.18 \quad, \quad S_{2}\left((F, E),\left(F_{1}, E\right)\right)=0$,

$S_{3}\left((F, E),\left(F_{1}, E\right)\right)=0.13, S_{4}\left((F, E),\left(F_{1}, E\right)\right)=0.27$,

$S_{5}\left((F, E),\left(F_{1}, E\right)\right)=0.20, S_{6}\left((F, E),\left(F_{1}, E\right)\right)=0.45$,

$S_{7}\left((F, E),\left(F_{1}, E\right)\right)=0.06$

Thus we have $S\left((F, E),\left(F_{1}, E\right)\right)=0.45<\frac{1}{2}$

Case II: Similarity between $(F, E)$ and $\left(F_{2}, E\right)$

$$
(F, E) \tilde{\cup}\left(F_{2}, E\right)=\left(P_{2}, E\right)
$$

$\begin{array}{cccccccc}\left(P_{2}, E\right) & e_{1} & e_{2} & e_{3} & e_{4} & e_{5} & e_{6} & e_{7} \\ y & (1,0) & (1,0) & (0.2,0) & (1,0) & (0.5,0) & (0.1,0) & (1,0) \\ n & (0.1,0) & (0.1,0) & (1,0) & (0.1,0) & (1,0) & (1,0) & (0.1,0)\end{array}$

$$
(F, E) \tilde{\cap}\left(F_{2}, E\right)=\left(Q_{2}, E\right)
$$

$$
\begin{array}{cccccccc}
\left(Q_{2}, E\right) & e_{1} & e_{2} & e_{3} & e_{4} & e_{5} & e_{6} & e_{7} \\
y & (0.7,0) & (0.9,0) & (0,0) & (0.6,0) & (0,0) & (0,0) & (0.8,0) \\
n & (0,0) & (0,0) & (0.2,0) & (0,0) & (0.3,0) & (0.7,0) & (0,0)
\end{array}
$$

The imprecise soft matrices corresponding to these two imprecise soft sets $\left(P_{2}, E\right)$ and $\left(Q_{2}, E\right)$ are given by,

$$
\begin{aligned}
& A_{2}=\left[\begin{array}{ccccccc}
(1,0) & (1,0) & (0.2,0) & (1,0) & (0.5,0) & (0.1,0) & (1,0) \\
(0.1,0) & (0.1,0) & (1,0) & (0.1,0) & (1,0) & (1,0) & (0.1,0)
\end{array}\right] \\
& B_{2}=\left[\begin{array}{ccccccc}
(0.7,0) & (0.9,0) & (0,0) & (0.6,0) & (0,0) & (0,0) & (0.8,0) \\
(0,0) & (0,0) & (0.2,0) & (0,0) & (0.3,0) & (0.7,0) & (0,0)
\end{array}\right]
\end{aligned}
$$
by,

The corresponding presence level matrices are given

$$
P L\left(A_{2}\right)=\left[\begin{array}{ccccccc}
1 & 1 & 0.2 & 1 & 0.5 & 0.1 & 1 \\
0.1 & 0.1 & 1 & 0.1 & 1 & 1 & 0.1
\end{array}\right]
$$




$$
P L\left(B_{2}\right)=\left[\begin{array}{ccccccc}
0.7 & 0.9 & 0 & 0.6 & 0 & 0 & 0.8 \\
0 & 0 & 0.2 & 0 & 0.3 & 0.7 & 0
\end{array}\right]
$$

We have,

$$
\begin{aligned}
& S_{1}\left((F, E),\left(F_{2}, E\right)\right)=0.64, S_{2}\left((F, E),\left(F_{2}, E\right)\right)=0.82, \\
& S_{3}\left((F, E),\left(F_{2}, E\right)\right)=0.17, S_{4}\left((F, E),\left(F_{2}, E\right)\right)=0.55, \\
& S_{5}\left((F, E),\left(F_{2}, E\right)\right)=0.20, S_{6}\left((F, E),\left(F_{2}, E\right)\right)=0.64, \\
& S_{7}\left((F, E),\left(F_{2}, E\right)\right)=0.06
\end{aligned}
$$

Thus we have $S\left((F, E),\left(F_{2}, E\right)\right)=0.82>\frac{1}{2}$

In view of our notion of similarity of two imprecise soft sets, we can conclude that the second ill person is possibly suffering from pneumonia.

\section{Conclusion}

Baruah has already established that two independent laws of randomness are necessary and sufficient to define a law of fuzziness. Secondly, the existing definition of complement of a fuzzy set is logically incorrect. He has introduced the theory of imprecise sets in which these two fuzzy set theoretic blunders are absent. Keeping in view, we have initiated the notion of imprecise soft sets, which is a hybrid model of Molodtsov's soft sets and Baruah's imprecise sets. Imprecise soft sets are different from Maji's fuzzy soft sets in the sense that in our work, we have replaced Zadeh's fuzzy sets with Baruah's imprecise sets. It is hoped that our work would help enhancing the study in fuzziness.

\section{Acknowledgment}

The authors would like to thank the anonymous reviewers for their careful reading of this paper and for their helpful comments which have improved this work.

\section{References}

[1] H. K. Baruah, "The Theory of Fuzzy Sets: Beliefs and Realities", International Journal of Energy, Information and Communications, Vol. 2, Issue 2, pp. 1-22, May 2011.

[2] H. K. Baruah, "An Introduction to The Theory of Imprecise Sets: The Mathematics of Partial Presence", Journal of Mathematical and Computational Sciences, Vol. 2, No. 2, pp. 110-124, 2012.

[3] D.A. Molodtsov, "Soft Set theory -First Result", Computer and Mathematics with Applications 37 (1999) 19-31.
[4] P. K. Maji and A.R. Roy, "Soft Set Theory", Computers and Mathematics with Applications 45 (2003) $555-562$.

[5] T.J. Neog and D. K. Sut, “ A New Approach To The Theory of Soft Sets", International Journal of Computer Applications, Vol 32, No 2,October 2011, pp 1-6.

[6] P.K.Maji, R.Biswas and A.R.Roy , "Fuzzy soft Sets", Journal of Fuzzy Mathematics, Vol 9, no.3, pp.-589-602, 2001.

[7] B. Ahmad and A. Kharal, "On Fuzzy Soft Sets", Advances in Fuzzy Systems, Volume 2009.

[8] T.J. Neog, D. K. Sut, “ On Fuzzy Soft Complement And Related Properties" International Journal of Energy, Information and Communications, Volume 3, Issue 1, February-2012, pp. 23-34 .

[9] T.J. Neog, D. K. Sut, "Theoty of Fuzzy Soft Sets from a new perspective", International Journal of Latest Trends in computing, Vol2, No 3, September 2011, pp. 439-450.

[10] H. K. Baruah, “Towards Forming A Field of Fuzzy Sets", International Journal of Energy, Information and Communications, Vol. 2, Issue 1, pp. 16-20, February 2011.

[11]H. K. Baruah, "Theory of Fuzzy Sets: The Case of Subnormality", International Journal of Energy, Information and Communications, Vol. 2, Issue 3, pp. 1-8, August 2011.

[12]H. K. Baruah, "In search of the root of fuzziness: The measure theoretic meaning of partial presence", Annals of Fuzzy Mathematics and Informatics, 2011.

\section{Authors' Profiles}

Tridiv Jyoti Neog (1980-) received his M.Sc. degree in Mathematics from Dibrugarh University, India, in 2004. He is a research scholar in the department of Mathematics, Faculty of Science, CMJ University, Shillong, Meghalaya, India. $\mathrm{He}$ was awarded "BINANDI MEDHI MEMORIAL AWARD" for being the best graduate in B.Sc. examination, 2001, under Dibrugarh University.

Dusmanta Kumar Sut $(1975-)$ received his M.Sc. degree in Mathematics from Dibrugarh University, Dibrugarh, India, in 2002 and his Ph.d in Mathematics from Dibrugarh University, India, in 2007. He is an assistant professor in the department of Mathematics, N. N. Saikia College, Titabor, India. His research interests are in Fuzzy Mathematics, Fluid dynamics and Graph Theory. 